\title{
Active Flow Control at the Rear End of a Generic Car Model Using Steady Blowing
}

\author{
R. Mestiri ${ }^{1}$, A. Ahmed-Bensoltane ${ }^{2}$, L. Keirsbulck ${ }^{3}$, F. Aloui $^{3 \dagger}$ and L. Labraga $^{3}$ \\ 1 LESTE, Ecole Nationale d'Ingénieurs de Monastir, Tunisie \\ 2 Ecole Supérieure des Techniques de l'Aéronautique, Alger, Algérie \\ 3 TEMPO, EA 4542, 59313 Valenciennes, Université Lille Nord de France \\ $\dagger$ Corresponding Author Email: fethi.aloui@univ-valenciennes.fr
}

(Received October 13, 2013; accepted November 18, 2013)

\begin{abstract}
Numerous aerodynamic designs of automotive vehicle have been made to reduce aerodynamic drag for lower fuel consumption. Indeed, automotive industry was primarily interested in the passive control based on the shape changes. But, as shape modifications are limited by several factors, this industry is recently more focused on active flow control. In this experimental investigation, the influence of continuous blowing along the sharp edge between the roof and the rear window is addressed. This actuation represents a new configuration based on a steady blowing tangentially to the surface of the rear window of the $25^{\circ}$ slanted Ahmed body model. The study was carried out in a wind tunnel at Reynolds numbers based on the model length up to $2.78 \times 10^{6}$. The actuation leads to a maximum drag reduction slightly upper than $10 \%$ obtained with a Reynolds number of $1.74 \times 10^{6}$ and a blowing velocity of $0.65 V_{0}$, where $V_{0}$ is the freestream velocity. Reductions between $6 \%$ and $7 \%$ were obtained for the other studied cases. These aerodynamic drag measurements were used to evaluate the actuator efficiency which reveals a maximum efficiency of 9 . Visualizations show that tangential steady blowing increase the separated region on the rear window and consequently disrupt the development of the counter-rotating longitudinal vortices appearing on the lateral edges of the rear window. It is also noted that the flow is reattached to the upper half of the rear window. As the actuation occurred directly on the recirculation region at the top of the rear window wall, the flow control was seen very effective.
\end{abstract}

Keywords: Ahmed body, Steady blowing, Control, Drag reduction

NOMENCLATURE

$C_{D} \quad$ drag coefficient

$C_{\mu} \quad$ steady momentum coefficient

$e^{\quad \text { blowing slit spacing }}$

$F_{D} \quad$ time averaged drag force

$K$ generic total pressure loss of the blowing system

$L \quad$ ahmed body length

$l^{*} \quad$ rear window length

$P_{c} \quad$ energy used to generate the control

$P_{e c} \quad$ energy saved through aerodynamic drag reduction

$\operatorname{Re}_{L} \quad$ Reynolds number based on the Ahmed body length

$S_{b} \quad$ blowing section
$S_{\infty} \quad$ Frontal surface of the Ahmed body

$V_{0} \quad$ upstream velocity

$V_{b} \quad$ blowing velocity

$V_{b, c} \quad$ critical blowing velocity

$V_{b, \max }$ maximum blowing velocity

$V_{b, s a t} \quad$ saturation blowing velocity

$W \quad$ Ahmed body width

$\Delta C_{D} \quad$ drag reduction

$\Delta F_{D} \quad$ drag force variation

$\rho \quad$ density of air

$\zeta \quad$ actuator efficiency

\section{INTRODUCTION}

Automotive industries meet several problems such as large consumption of fuel and pollutant emissions. To reduce these embarrassing effects and improve the vehicle performance, flow control pro- cedures are adopted. The general objective of the control is to move separations towards the rear part of the vehicle, to suppress or at least to reduce the longitudinal vortex structures, or to reduce the width of the wake (Tounsi, Fourrié, Oualli, Keirsbulck, Hanchi, and Labraga 2012). The flow 
over a road vehicle is very complex, unsteady and $3 \mathrm{D}$ and many studies have been carried out on the airflow control at the rearward of a simplified geometry of Ahmed body. Passive (Fourrié et al. (2011), Beaudoin and Aider (2008), Bruneau et al. (2010), Depeyras (2009), Fiedler and Fernholz (1993), Gilliéron (2002)) and active (Joseph et al. (2012), Leclerc et al. (2006), Brunn and Nitsche (2006), Rouméas et al. (2006), Henning et al. (2008), Rouméas et al. (2008)) control techniques have been studied. Few studies have been devoted to the active control with continuous blowing, here the main ones are briefly reviewed. Rouméas et al. (Rouméas, Gilliéron, and Kourta 2009) analyzed numerically the influence of the blowing on the near-wake flow topology of square-back geometry. The blowing slots were distributed around the base periphery at an inclination with respect to the vertical plane of $45^{\circ}$. In their numerical study (Rouméas, Gilliéron, and Kourta 2009), the authors obtained a maximum drag reduction of $29 \%$ with a blowing velocity of $1.5 \mathrm{~V}_{0}$. However, this significant drag gain corresponded to a very low efficiency (efficiency is defined as the ratio between the save power due to drag reduction and supplied pneumatic power) while the largest efficiency which is equal to 7 was obtained with $V_{b}=0.5 V_{0}$ and when the drag reduction is of $20 \%$. Authors (Rouméas, Gilliéron, and Kourta 2009) showed a reduction in the wake section, a total pressure loss reduction in the wake and an increase of the static pressure in the rear part of the geometry. Lehugeur et al. (Lehugeur et al. (2006), Lehugeur et al. (2008)) conducted numerical and experimental studies on the flow control by steady blowing normal to the lateral face of the Ahmed body having a rear window angle of $25^{\circ}$. Continuous blowing was ensured via two slots along the two lateral edges of the slanted rear window of the Ahmed body and leading to longitudinal vortex breakdown. The authors (Lehugeur, Gilliéron, and Ivanic 2006) showed through their numerical simulation a drag reduction of $6 \%$ and an efficiency of 1.5. Their experimental investigation (Lehugeur, Gilliéron, and Bobillier 2008) was focused on a better understanding of the mechanism of the vortex breakdown through the vorticity fields and the analysis of the azimuthal and longitudinal velocities. Longitudinal vortex breakdown was obtained for high blowing velocity $\left(V_{b}=1.5 V_{0}\right)$. Another active control technique using steady blowing was applied by Aubrun et al. (Aubrun, McNally, Alvi, and Kourta 2011), the actuator consisted of an array of steady microjets placed $6 \mathrm{~mm}$ downstream of the upper sharp edge of the $25^{\circ}$ slanted rear window. Authors (Aubrun, McNally, Alvi, and Kourta 2011) showed that the location of microjets was very effective in controlling the separation bubble at the rear window. They noted a maximum drag reduction of $14 \%$ when the upstream velocity was $40 \mathrm{~m} / \mathrm{s}$ and the momentum coefficient, as they computed, was $2.6 \%$. They also found that the separation was completely suppressed for the higher blowing flow rate, whereas it was just delayed for lower blowing rate.

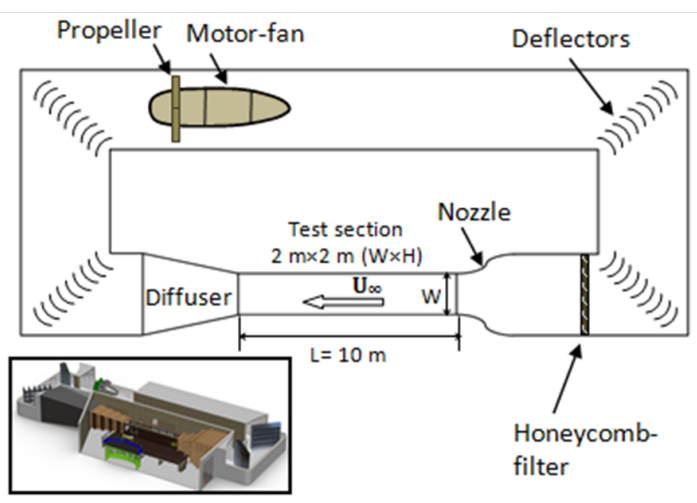

\section{Fig. 1. Diagram of the closed-circuit subsonic wind tunnel: Sketch showing the main components.}

The present study is the first experimental approach on continuous blowing, based on the numerical results of Rouméas et al. (Rouméas, Gilliéron, and Kourta 2009). The aim of this work is to reduce the drag and offer more explanation of the drag reduction mechanism via drag measurements and surface visualizations. The evolution of the drag reduction versus the blowing velocity will be highlighted. We will show through our new blowing configuration that the actuator ensures an important drag reduction and a high efficiency. So, we can say that flow control using fluidic actuator based on blowing offers interesting prospect in term of energy.

\section{EXPERIMENTAL SET UP AND METH- ODS}

The experiments were conducted in a closed-circuit subsonic wind tunnel of the TEMPO laboratory. The test section is $10 \mathrm{~m}$ long with a $2 \times 2 \mathrm{~m}^{2}$ square cross section (Fig. 1). The maximum freestream velocity is equal to $60 \mathrm{~m} / \mathrm{s}$ in the test section. The upstream conditions are as follows: the incoming boundary layer height is about $3 \times 10^{-2} \mathrm{~m}$, the blockage ratio is about $3 \%$ and the turbulence intensity is about $0.5 \%$ in the vacuum test section. The bluff-body used in our study is a full-scale Ahmed body with a $25^{\circ}$ rear slant angle (Ahmed et al. (Ahmed, Ramm, and Faltin 1984)) as shown in Fig. 2. The model is installed in the wind tunnel section on a 6-axis aerodynamic balance at a height of $79 \mathrm{~mm}$ from the subfloor of the wind tunnel using 4 cylinders connected to the aerodynamic balance. The time-averaged drag $\left(F_{D}\right)$ was measured 
by the external aerodynamic balance and the drag coefficient was calculated as follows:

$C_{D}=\frac{F_{D}}{1 / 2 \rho V_{0}^{2} S_{\infty}}$

where $S_{\infty}$ is the projected area of the car model.

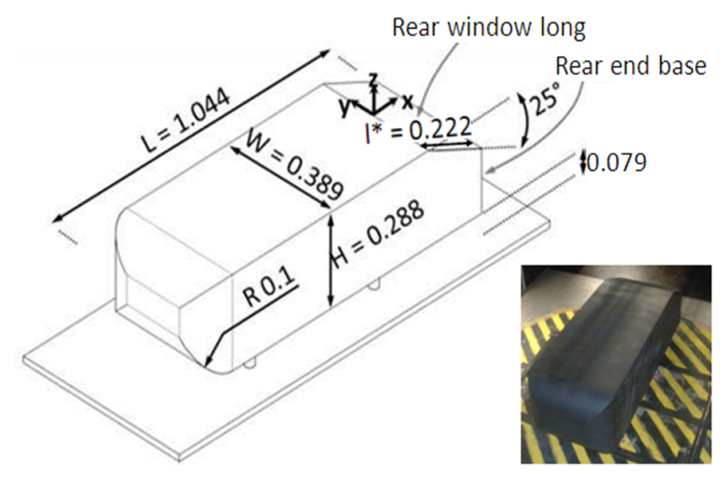

Fig. 2. Geometry of the Ahmed car model (dimensions in m), Ahmed et al. (Ahmed, Ramm, and Faltin 1984).

The absolute uncertainty on the drag coefficient depends on both the tolerance of the load cell provided by the manufacturer and the self sustain stability. Drag measurements are done with airflow Reynolds number based on the model length up to $R e_{L}=2.78 \times 10^{6}$. Acquisition is made for the voltage values and then these voltages are converted in drag force using a calibration procedure. The relative uncertainty on the drag coefficient is about $1.6 \%$ for the highest Reynolds number $\left(2.78 \times 10^{6}\right)$. The flow control used in this present study is based on a continuous blowing provided by a slit actuator (Fig. 3). The blowing slit length is equal to the model width (W) and its spacing (e) is of 1 $\mathrm{mm}$. The metal box of the blowing device was filled with spherical balls in order to homogenize the flow along the slit exit. The pressure inlet could be adjusted using a valve and evaluated through a BROOK mass flow controller. The actuator is flush mounted on the roof surface of the Ahmed body. Consequently, the actuation consists of tangential blowing along the upper edge of the rear window as schematically presented in Fig. 3(a), with a blowing velocity range of 0 to $22 \mathrm{~m} / \mathrm{s}$.

Wall friction lines are visualized on the rear end window using a mixture of paraffin oil, oleic acid and titanium oxide. The proportions were adapted to obtain clear friction patterns. An approximately $0.5 \times 10^{-3} \mathrm{~m}$ thick layer of the white viscous mixture was applied on the rear window, previously painted black. The images were taken using a numerical camera with a $35 \mathrm{~mm} f / 3.5$ objective.

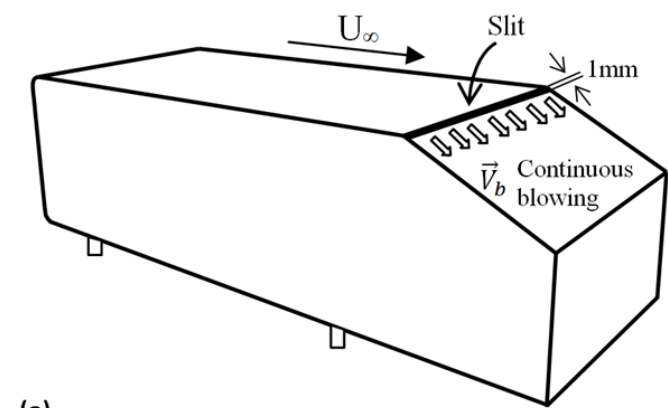

(a)

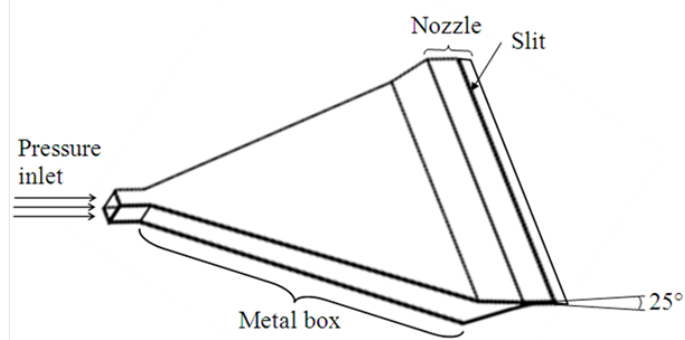

(b)

Fig. 3. Description of the control device: (a) overview and (b) design of blowing actuator nozzle.

\section{RESULTS AND DISCUSSION}

\subsection{Actuator blowing: spatial distribution and baseline flow}

The blowing set up was characterized before being integrated in the model using Pitot tube measurements. The Pitot tube was moving along the slot length and it was fixed to a support enabling it to be parallel to the flow direction during the system movement (fig. 4(a)). The blowing velocity measurements, made at $4 \mathrm{~mm}$ downstream of the slot, reveal a bearing (Fig. 4(b)). The maximum blowing velocity Vbmax is close to $22 \mathrm{~m} / \mathrm{s}$. The mean blowing velocity flow homogeneity is $3 \%$ along a transverse distance of $200 \mathrm{~mm}$, from $y=74.5 \mathrm{~mm}$ to $y=+125.5 \mathrm{~mm}$. It is an acceptable value confirming that the used blowing system can be considered to be a continuous blowing system ensuring a uniform blowing along the slot length.

Fig. 5 shows that the drag coefficient decreases with the Reynolds number. This evolution is entirely consistent with previous works performed on the study of the flow at the rear of a bluff body and the baseline drag coefficients are very close to the coefficients found by Thacker (Thacker 2010).

\subsection{Continuous blowing effects on drag reduc- tion and flow topology}

The relative aerodynamic drag reduction $\Delta C_{D}$ is calculated from the drag value measured before actuation and that measured when the control is actu- 


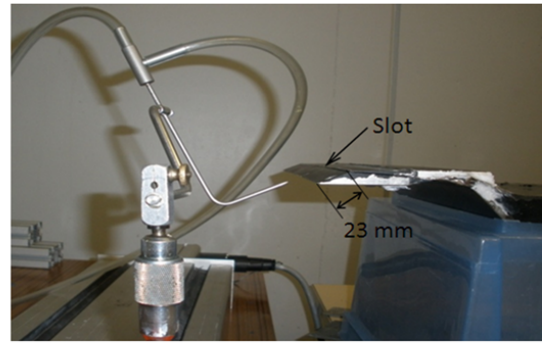

(a)

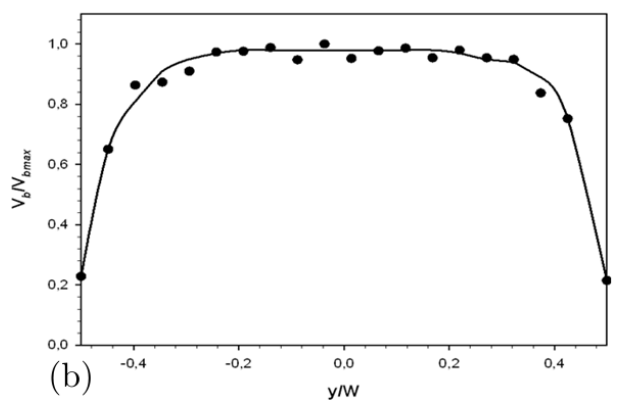

Fig. 4. Actuator flow characterization: (a) velocity measurement set-up and (b) typical blowing velocity profile along the slot length

(W).

ating, as follows:

$\Delta C_{D}(\%)=\frac{C_{D(\text { withcontrol })}-C_{D(\text { withoutcontrol })}}{C_{D(\text { withoutcontrol })}} \times 100($

Drag measurements are conducted for Reynolds numbers from $1.74 \times 10^{6}$ to $2.78 \times 10^{6}$ (corresponding respectively to freestream velocities between $25 \mathrm{~m} / \mathrm{s}$ and $40 \mathrm{~m} / \mathrm{s}$ ).

To quantify the interaction between the steady blowing and the freestream, we define the momentum coefficient $C_{\mu}$ as the ratio of the momentum injected by the steady jet and the momentum of the upstream airflow (see Eq. 3).

$C_{\mu}=\frac{S_{b}}{S_{\infty}}\left(\frac{V_{b}}{V_{\infty}}\right)^{2}$

where $S_{b}$ is the blowing section $(W \times e)$

Fig. 6 illustrates the evolution of the rate of the drag change as function of $C_{\mu}$ for four different Reynolds numbers. The continuous blowing is initially not effective for low jet velocities and exhibits strong overshoot. The drag is almost constant or increases with the blowing onset. However, above a critical momentum coefficient $C_{\mu, c}$ the actuation reduces the drag and the efficiency of the actuator becomes obvious for all these cases that are characterized by maximum drag gain between
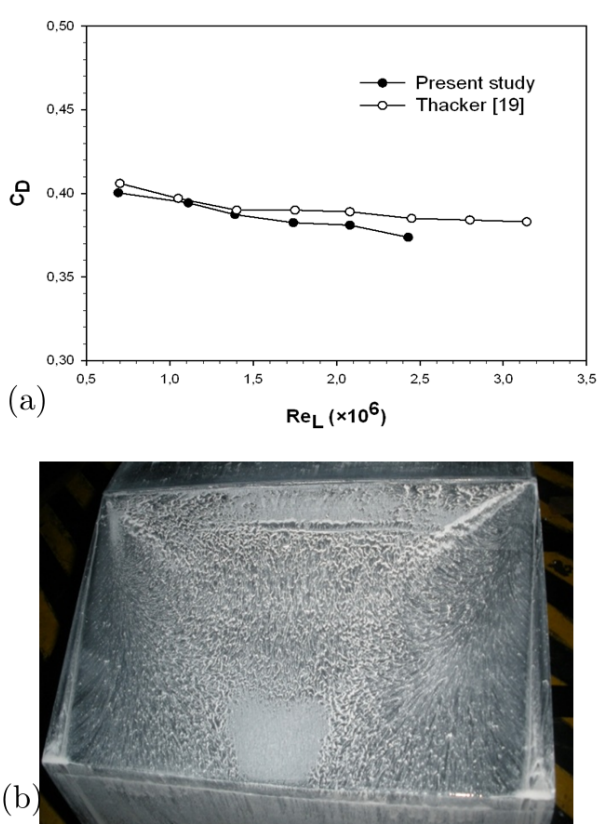

Fig. 5. (a) Drag coefficient versus the Reynolds number and $(b)$ the baseline flow visualization performed in the present study for $R e_{L}=2.08 \times 10^{6}$.

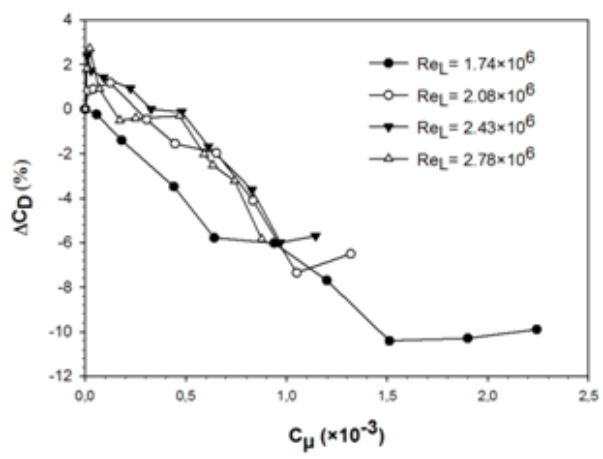

Fig. 6. Drag reduction as function of the steady momentum coefficient for different $R e_{L}$.

6 and $10.4 \%$. The critical value $C_{\mu, c}$ above which the actuator becomes effective is different for each upstream velocity. We note that critical blowing increases with the incoming velocity, the greater the upstream velocity the greater the required input power. Then, the aerodynamic drag continues to decrease nearly monotonically with increasing flow velocity through the slot until reaching the saturation point $C_{\mu, s a t}$ beyond which the drag no longer decreases with the increase of the actuator flow. Depending on the Reynolds number, the minima seen on Fig. 6 correspond to different drag reduction rates and different momentum coefficients. For $\operatorname{Re}_{L}=1.74 \times 10^{6}$, a minimum is obtained for $\Delta C_{D}=10.4 \%$ and $C_{\mu, \text { sat }}=1.5 \times 10^{-3}$ (the corresponding blowing velocity is $0.65 V_{0}$ ). For the remaining cases, minima are obtained with $\Delta C_{D}$ be- 
tween $6 \%$ and $7.4 \%$ when the saturation momentum is nearly $10^{-3}$ (corresponding to a blowing velocity almost $0.5 V_{0}$ ). These results are similar to those obtained by Aubrun et al. (Ahmed, Ramm, and Faltin 1984). The drag evolution illustrated in their work (Ahmed, Ramm, and Faltin 1984) showed that the drag reduction versus momentum coefficient had the same shape and the same definition of the momentum coefficient thresholds (as $C_{\mu, c}$ and $C_{\mu, s a t}$ ). However, as the authors (Ahmed, Ramm, and Faltin 1984) have studied an Ahmed body model of smaller scale (0.7) and have calculated the momentum coefficient differently, for the same studied airflow velocities, our curves and theirs do not have the same scale and therefore, a gap exists between the present results and those found by Aubrun et al. (Ahmed, Ramm, and Faltin 1984).

Energetic efficiency of the steady blowing actuation is calculated in order to evaluate the energetic cost. The following efficiency expression is proposed by Roumás et al. (Rouméas, Gilliéron, and Kourta 2009):

$\zeta=\frac{P_{e c}}{P_{c}}=\frac{2 \Delta F_{D} V_{0}}{K \rho V_{b}^{3} S_{b}}, P_{e c}=\Delta F_{D} V_{0}, P_{c}=\frac{K \rho V_{b}^{3} S_{b}}{2}(4)$

where $P_{e c}$ and $P_{c}$ represent respectively the energy saved through aerodynamic drag reduction and the energy used to generate the control, $K$ is sthe generic total pressure loss of the blowing system. The total pressure loss coefficient $K$ of the blowing system is assumed to be equal to 5 (Rouméas, Gilliéron, and Kourta 2009).

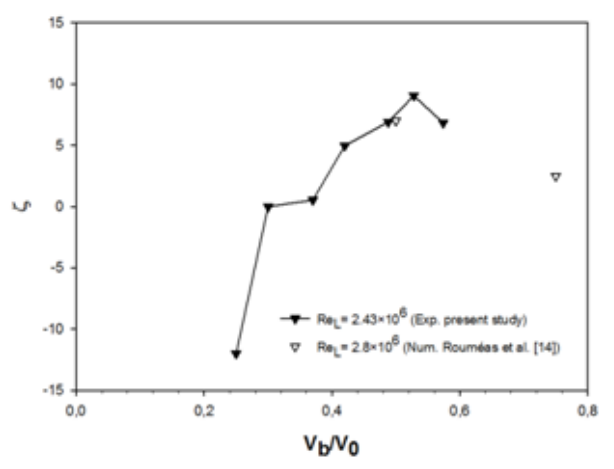

Fig. 7. Actuator efficiency versus the blowing velocity: comparison between the present experimental results and the numerical results of Rouméas et al. (Rouméas, Gilliéron, and Kourta 2009).

The control is considered efficient when the value of $\zeta$ is equal to or greater than $\zeta_{c}=1$ (Rouméas, Gilliéron, and Kourta 2009). The illustrated results in Fig. 7 show a negative efficiency for low blowing

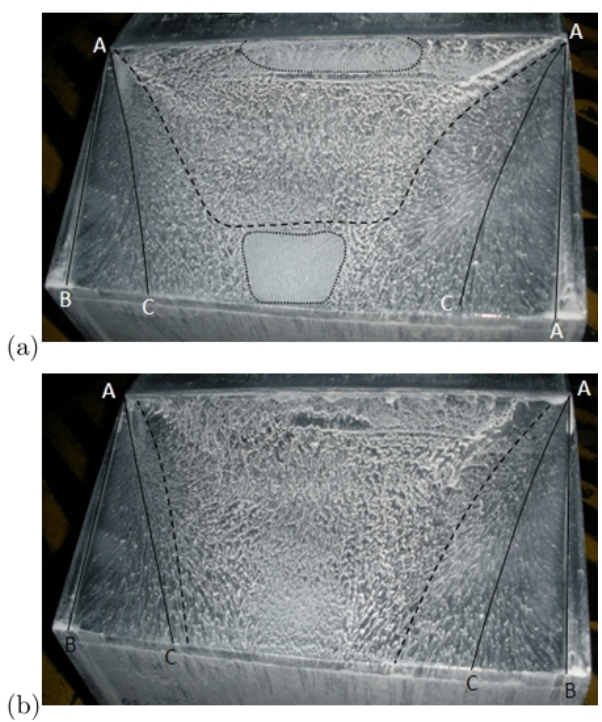

Fig. 8. Cartography of the friction lines on the rear window $\left(\operatorname{Re}_{L}=2.08 \times 10^{6}, V_{0}=30 \mathrm{~m} / \mathbf{s}\right.$, $V_{b}=16.5 \mathrm{~m} / \mathbf{s}$ ), (a): without control and (b) with control

velocities, matching with the previously indicated overshoot. We observe that the actuation is not efficient for $V_{b} / V_{0}<0.4$. Maximum efficiency is obtained when the blowing velocity is almost equal to $0.5 V_{0}$. Illustrated numerical results of Rouméas et al. (Rouméas, Gilliéron, and Kourta 2009) show, for a close Reynolds number, that the highest efficiency is obtained when $V_{b} / V_{0}=0.5$, which is similar to the present study. As aerodynamic drag measurements clearly show the effectiveness of the blowing control in reducing drag, flow mapping is done in order to understand the flow modifications leading to drag reduction and to determine the differences in flow field in response to flow control. The oil film visualizations show the effect of the steady jet on the separated bubble and the longitudinal vortex. The rear window is covered with thin superposed sheets of viscous solution. The patterns are identified with letters and lines in order to provide a better visibility of the flow vortex progression at the rear end of the Ahmed body; this adopted identification is previously used by Spohn et al. (Spohn and Gilliron 2002). Line AB is the separation line of the secondary vortex, $\mathrm{AC}$ is the attachment line of the main vortex, the dotted line corresponds to the limit of the separated region over the rear window. The longitudinal vortex is represented by the triangle ABC. Fig. 89 illustrate the surface rear window visualizations for $R e_{L}=2.08 \times 10^{6}$ and $R e_{L}=2.43 \times 10^{6}$, corresponding to freestream velocities of $30 \mathrm{~m} / \mathrm{s}$ and 35 $\mathrm{m} / \mathrm{s}$. Surface visualizations give binary information of the separated or attached nature of the boundary layer. Generally, when the boundary layer is 
separated, the oil tracers show a random behavior and there is not a clear direction of the flow. Mapping of baseline flow (Fig. 8 (a) and Fig. 9 (a)) presents two intact areas which are not affected by the flow. These areas are represented by small dotted lines, a top area and a low area. These two areas are not affected by the flow due to a detachment mechanism of the separated bubble. The separation bubble changes spatially along the rear window length from attached flow to a fully separated one. When the upstream velocity increases, we see that the area of fully separated flow becomes less extended. For the baseline flow, the extension of the separated zone is between $1 / 2$ and $2 / 3$ of the rear window width and we observe that the separated bubble size increases with the Reynolds number. For Reynolds number of $2.08 \times 10^{6}$, the separated region is spread along nearly the half of the rear window length and a larger surface is occupied for the larger Reynolds number. Cartography of the controlled flow is illustrated by photos showing the control effect on the separated region (Fig. 8 (b) and Fig. 9 (b)). We note that intact areas no longer exist and that the friction lines reveal a more attached flow to the rear window wall. The observed directed friction lines show that the spatial detachment mechanism is suppressed and that the span of the resulting attachment depends on the freestream velocity. Indeed, the control pushes the longitudinal vortex towards the rear window edge and then reduces their impact area on the rear window. The continuous blowing weakens the longitudinal vortex. For $R e_{L}=2.43 \times 10^{6}$, the streamlines are well directed at the top of the rear window, whereas we see in the down part a random motion showing a recirculation region (Fig. 9 (b)).

\section{Conclusion}

The present work has highlighted the efficiency of the continuous blowing to reduce the drag and to control the separation bubble located on the rear window of the Ahmed body. The main result concerning this first experimental approach, based on the numerical works of Rouméas et al. (Rouméas, Gilliéron, and Kourta 2009), is a drag coefficient reduction of $6-10.4 \%$, depending on the Reynolds number. The drag reduction evolution shows that the optimum momentum coefficient defined as the coefficient corresponding to the maximum drag reduction is not necessarily the maximum momentum coefficient. The optimal coefficient is almost $10^{-3}$ for all the studied free airstream velocities. The efficiency investigation reveals that the actuator is more effective when the blowing velocity is close to $0.5 V_{0}$ and that the efficiency is close to 9 for the studied Reynolds number range. The controlled flow visualizations made for the maximum drag reduction clearly show that the separation is

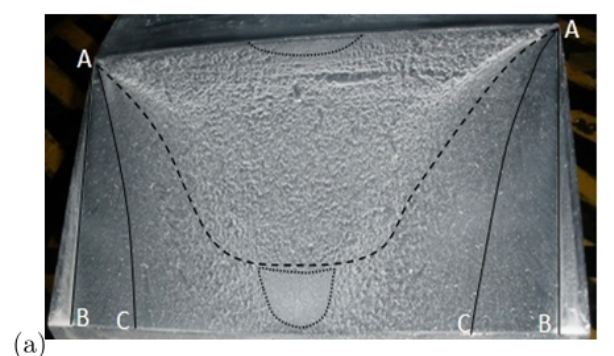

(a)

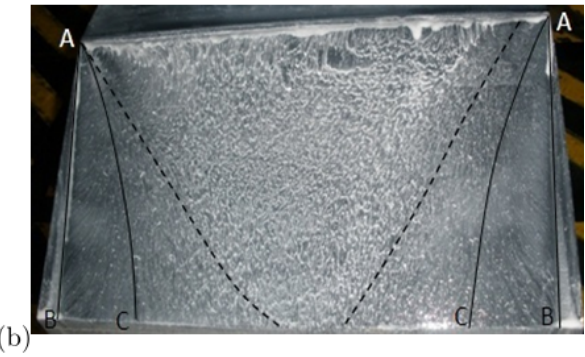

Fig. 9. Cartography of the friction lines on the rear window $\left(R e_{L}=2.43 \times 10^{6}, V_{0}=35 \mathrm{~m} / \mathbf{s}\right.$, $V_{b}=18.5 \mathrm{~m} / \mathrm{s}$ ), (a): without control and (b) with control.

strongly three-dimensional. Visualizations also reveal the signature of the steady streamwise "corner" vertical structure at both edges of the rear window for both controlled and uncontrolled cases. After actuation the flow seems to be reattached to the rear window wall, the continuous blowing mechanism reattaches the flow at the top of the rear window and then pushes the separation bubble down. Our fluidic actuator was shown effective to control the flow at the rear end of the Ahmed body. Future studies include a detailed characterization using high-resolution PIV and unsteady pressure information on the rear window in order to examine the control influence on the unsteady flow properties and the three-dimensional aspects of the controlled topology.

\section{ACKNOWLEDGMENTS}

The present research work has been supported by international Campus on Safety and Intermodality in Transportation, the Nord-Pas-de-Calais Region, the European Community, the Regional Delegation for Research and Technology, the Ministry of Higher Education and Research, and the National Center for Scientific Research. The authors gratefully acknowledge the support of these institutions.

IN MEMORY OF OUR COLLEAGUE, PROFESSOR LARBI LABRAGA

This research topic, on the boundary layer separation and its control, was supervised at the TEMPO laboratory of the University of Valenciennes, by our colleague, Professor Larbi LABRAGA, co-author of 
the current study, who left us in November 2013 after the submission of this paper, after a long illness. We would like firstly to him a very great tribute. Professor Larbi LABRAga was an excellent colleague, very serious, scientifically very curious and very human. With him, we lost more than just a colleague, but a very dear friend.

\section{REFERENCES}

Ahmed, S., G. Ramm, and Faltin (1984). Some salient features of the time-averaged ground vehicle wake. SAE Paper 840300, Detroit, USA.

Aubrun, S., J. McNally, F. Alvi, and A. Kourta (2011). Separation flow control on a generic ground vehicle using steady microjet arrays. Exp. Fluids 51, 1177-1187.

Beaudoin, J.-F. and J.-L. Aider (2008). Drag and lift reduction of a $3 \mathrm{~d}$ bluff-body using flaps. Exp. Fluids 44(4), 491-501.

Bruneau, C., E. Creusé, D. Depeyras, P. Gilliéron, and I. Mortazavi (2010). Coupling active and passive techniques to control the flow past the square back ahmed body. Computers \& Fluids 39, 1875-1892.

Brunn, A. and W. Nitsche (2006). Active control of turbulent separated flows over slanted surfaces. International Journal of Heat and Fluid Flow 27, 748-755.

Depeyras, D. (2009). Contrôles actifs et passifs appliqués l'aérodynamique automobile. $P h . D$. thesis, Bordeaux University, Bordeaux, FRANCE.

Fiedler, H. and H. Fernholz (1993). On management and control of turbulent shear flows. Program Aerospace Sci. 73(11), 287-300.

Fourrié, G., L. Keirsbulck, L. Labraga, and P. Gilliéron (2011). Bluff-body drag reduction using a deflector. Exp. Fluids 50, 385-395.

Gilliéron, P. (2002). Contôle des écoulements appliqué à l'automobile : état de l'art. Mécanique et Industrie 3(6), 515-524.

Henning, L., R. Becker, G. Feuerbach, R. Muminovic, R. King, R. Brunn, and W. Nitsche (2008). Extensions of adaptive slope-seeking for active flow control. Proc. IMechE, Part I: J. Systems and Control EngineeringE 222, Special Issue Paper 309.
Joseph, P., X. Amandolèse, and J.-L. Aider (2012). Drag reduction on the $25^{\circ}$ slant angle ahmed reference body using pulsed jets. Exp. Fluids 52, 1169-1185.

Leclerc, C., E. Levallois, Q. Gallas, P. Gilliéron, and A. Kourta (2006). Phase locked analysis of a simplified car geometry wake flow control using synthetic jet. Proc. FEDSM2006, ASME Joint U.S. European Fluids Engineering Summer Meeting, Miami, Florida.

Lehugeur, B., P. Gilliéron, and P. Bobillier (2008). Contrôle des structures tourbillonnaires longitudinales dans le sillage d'une géométrie simplifiée de véhicule automobile, approche expérimentale. Mécanique Industries 9, 533-541.

Lehugeur, B., P. Gilliéron, and T. Ivanic (2006). Contribution de l'éclatement tourbillonnaire à la réduction de la traînée des véhicules automobiles : approche numérique. Comptes Rendus Mcanique 334, 368-372.

Rouméas, M., P. Gilliéron, and A. Kourta (2006). Separated flows around the rear window of a simplified car geometry. Journal of Fluid Engineering $130(2)$.

Rouméas, M., P. Gilliéron, and A. Kourta (2008). Drag reduction by flow separation control on a car after body. Int. J. Numer. Meth. Fluids.

Rouméas, M., P. Gilliéron, and A. Kourta (2009). Analysis and control of the near-wake flow over a square-back geometry. Computers \& Fluids 38, 60-70.

Spohn, A. and P. Gilliron (2002). Flow separation generated by simplified geometry of an automotive vehicle. IUTAM Symposium on Unsteady Separated Flows, Toulouse, France.

Thacker, A. (2010). Contribution expérimentale à l'analyse stationnaire et instationnaire de l'écoulement à l'arrière d'un corps de faible allongement. Ph. D. thesis, Orléans University, FRANCE.

Tounsi, N., G. Fourrié, H. Oualli, L. Keirsbulck, S. Hanchi, and L. Labraga (2012). Volumetric 3component velocimetry measurements of the flow field on the rear window of a generic car model. Thermal Science 16(1), 313-320. 